\section{Iransfer of responses to open and closed shapes in discrimination learning by cats*}

\author{
J. M. WARREN \\ Pennsylvania State University, University Park, Pennsylvania 16802
}

Two sets of 32 cats each were matched for performance in learning to discriminate an open and a closed shape and were then trained on a transfer task. The consistent groups were rewarded for choosing the same class of shape (open or closed) in learning and transfer. The inconsistent groups were rewarded for choosing the open figure in one task and the closed figure in the second, or vice versa. After learning the transfer task, all of the Ss relearned the original discrimination task under the same conditions that prevailed in initial training. The inconsistent groups made more errors on both the transfer and retention problems; both these differences were significant at the $0.1 \%$ level of confidence. These results and those obtained on preference tests support the hypothesis that cats classify shapes as open or closed in terms of perimeter and number of sides.

Cats appear to classify visual shapes as "open" or "closed" on the basis of perimeter and number of sides. They make many more errors in learning to discriminate two closed figures (e.g., circle and triangle) or two open figures (e.g., $U$ and $I$ shapes) than in learning to discriminate heterogeneous pairs of one open and one closed figure (e.g. circle vs $U$, or triangle vs $I$ ) (Derdzinski \& Warren, 1969). Generalization tests (Warren \& McGonigle, 1969b) indicate that cats often respond to closed shapes, with few sides and/or a low perimeter:area ratio, as equivalent to a circle, and to open shapes, with many sides and/or a high perimeter :area ratio, as equivalent to an I shape.

The present experiment was designed to provide additional tests of the hypothesis that cats classify visual shapes as open or closed in terms of perimeter and number of sides. Groups of Ss were initially trained to discriminate between an open and a closed form, and then trained on a second discrimination with two different shapes. Half the cats (the consistent groups) were reinforced for choosing an open or closed form in both tasks, and half the $\mathrm{S}_{\mathrm{s}}$ (the inconsistent groups) were required to choose an open figure in the first task and a closed figure in the second, or vice versa. It was anticipated that if cats categorized shapes as open and closed on the basis of perimeter and number of sides, the consistent Ss would make significantly fewer errors than would the inconsistent $S$ s on the transfer task.

Additional tests of the hypothesis were provided by comparisons of the performance of the consistent and inconsistent groups in relearning the original discrimination task and on a

*This research was supported by Grant MH 04726 from National Institute of Mental Health, U.S. Public Health Service. series of generalization tests. On the hypothesis advocated here, one would expect less interference and hence superior retention and stronger preferences from the groups consistently reinforced for responding to open or closed figures than from the groups that learned to select the open figure in one pair and the closed figure in the second pair presented in the first two discrimination tasks.

\section{METHOD \\ Subjects}

Sixty-four laboratory-reared mongrel kittens, aged 120 days at the start of adaptation training, participated in this experiment. None of the Ss had served in any previous experiment.

\section{Apparatus and Stimuli}

The discrimination apparatus was $31 \mathrm{~cm}$ high and painted gray throughout. A $27 \times 54 \mathrm{~cm}$ startbox with opaque and transparent doors in series opened into a 40-cm-deep choice area, beyond which were two $30 \mathrm{x}$ $42 \mathrm{~cm}$ response compartments separated by an opaque wall. All the discriminanda used in this experiment were black wooden objects with areas of $80 \mathrm{~cm}^{2}$, cut from 2-cm-thick lumber and supported upright in the vertical plane by concealed braces. The shapes used in the learning, transfer, and retention phases of the experiment are illustrated in Fig. 2, and the stimuli used in the generalization tests are shown in Fig. 3. The discrimination apparatus and stimuli were illuminated by two $100-W$ bulbs in a ceiling fixture approximately $1.5 \mathrm{~m}$ above the apparatus.

\section{Procedure}

A trial began when $E$ raised the opaque door of the startbox. The transparent door was raised 3 sec later, and $\mathbf{S}$ was allowed to enter one of the response compartments. If the cat responded to the correct object, it was allowed to approach and to eat the reward (pork kidney or tuna fish) concealed behind the stimulus. If $S$ responded to the incorrect object, it was allowed to inspect it but was not permitted to correct the error. The position of the rewarded stimulus was varied from trial to trial according to the Gellermann sequences. The cubicle containing the apparatus in which the cats were tested was so thoroughly permeated with the odors of kidney and tuna that the small quantities of these foods used as the reward on individual trials provided no useful cues for localizing the correct stimulus on the basis of olfactory cues.

\section{Adaptation}

No stimulus objects were present in this phase of training; a $1 / 4-i n$. cube of kidney was presented on the floor of each goalbox, and $\mathrm{S}$ was allowed to take one piece of meat on each trial. The kittens were trained for 25 trials a day until they completed 25 trials in less than $10 \mathrm{~min}$, usually in a single session.

\section{Preference Test 1}

Two groups of 32 kittens each were tested with either a circle and a $U$ or a triangle and an I. The positions of the open and closed figures varied between trials in a balanced irregular sequence, all choices were reinforced, and $\mathbf{E}$
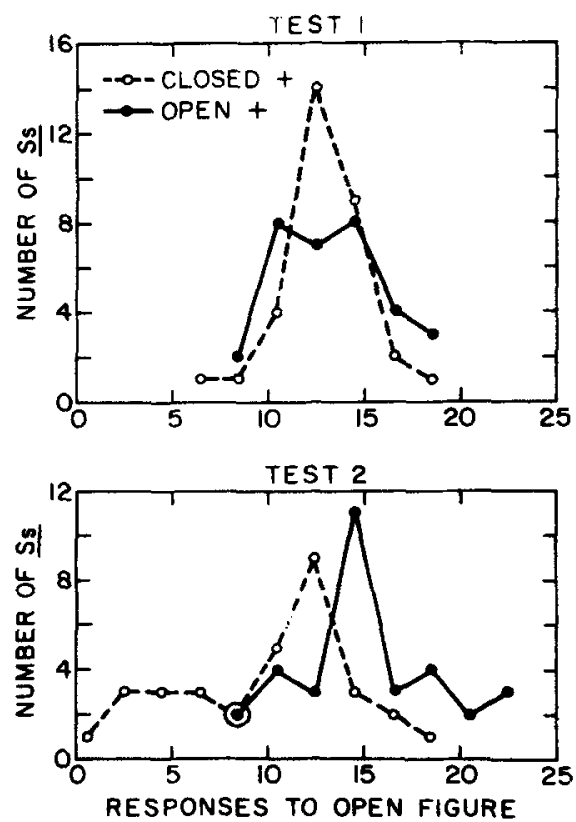

Fig..1. Distributions of individual Ss' responses to the open figure on 25-trial preference tests before (Test 1) and after (Test 2) discrimination training. 

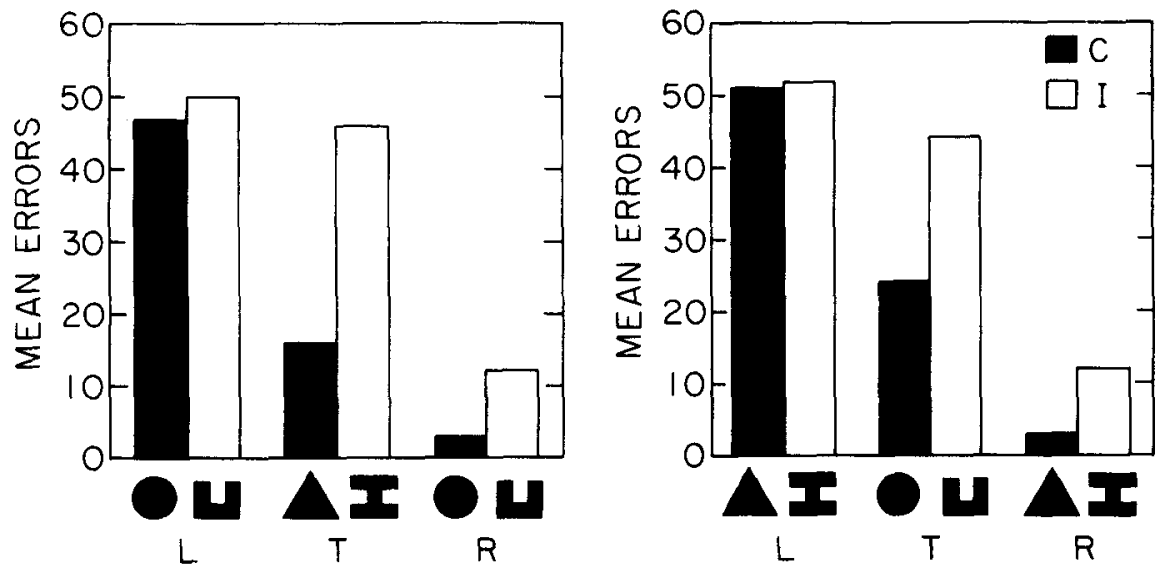

Fig. 2. Shapes used in learning (L), transfer ( $T$ ), and retention ( $R$ ) tasks and mean errors to criterion for consistent and inconsistent groups.

recorded the stimulus and side chosen on each of 25 trials.

\section{Learning}

The groups tested with each pair of figures were divided into subgroups, matched in terms of their preference for the open and closed figure in each pair to form four groups of 16 animals that were trained on the circle ${ }^{+}$vs $\mathrm{U}^{-}$, the $\mathrm{U}^{+}$vs circle-, the triangle ${ }^{+}$vs $\mathrm{I}^{-}$, or the $\mathrm{I}^{+}$vs triangle - discriminations, with 25 noncorrection trials per day, to a criterion of 10 consecutive correct responses in a single session.

\section{Preference Test 2}

On the day after reaching criterion on the initial discrimination, all $S s$ were tested for preferences with the pair of stimuli that they had not previously encountered. The conditions of the second preference test were identical with the first, except for the change in stimuli.

\section{Transfer}

Each of the four training groups was divided again into two groups of eight animals, matched in terms of the average number of errors to criterion in original learning. Four of these matched subgroups (the consistent groups) were required to choose the open or closed figure in transfer training in a manner that was consistent with their prior training. The inconsistent groups were forced to reverse their responses between the learning and transfer phases, being successively reinforced for choices of the open and closed figures, or vice versa, in the learning and transfer stages. The criterion of transfer learning was 10 consecutive correct responses in a single session.

\section{Retention}

All Ss were retrained to criterion on the discrimination they had initially learned, with the stimuli and reinforcement contingencies exactly as in the learning phase. This entailed a reversal of the responses to open and closed stimuli acquired in transfer training by the inconsistent but not by the consistent groups.

\section{Generalization Tests}

Half the Ss, four cats from each of the eight subgroups described above, were used in this phase of testing, which required 4 days. On each day, $\mathbf{S}$ was retrained to criterion on the retention problem with differential reinforcement and then given 25 test trials with reinforcement for responses to either stimulus in the four pairs shown in Fig. 3. The position of the objects was varied from trial to trial in accordance with the Gellermann sequences. The order in which the four pairs were presented to the members of each subgroup was counterbalanced by means of a Latin-square design to permit unequivocal estimation of the significance of repeated tests and of possible interpair differences in preferential responses.

\section{RESULTS}

Preference Tests

The results of the preference tests given before (Test 1) and after (Test 2) initial discrimination training with differential reinforcement are summarized in Fig. 1, which shows the distribution of responses to the open figure by the groups that were rewarded for choosing the open and closed figures in original learning. The results obtained in Preference Test 1 indicate that the groups were adequately matched and that neither group had a marked preference for the open or closed figures. The distributions are, however, quite dissimilar on Test 2, after training with differential reinforcement. The cats rewarded for choosing the closed figure in discrimination training selected the open shape on $52 \%$ of the trials in Test 1 , and on $40 \%$ of the

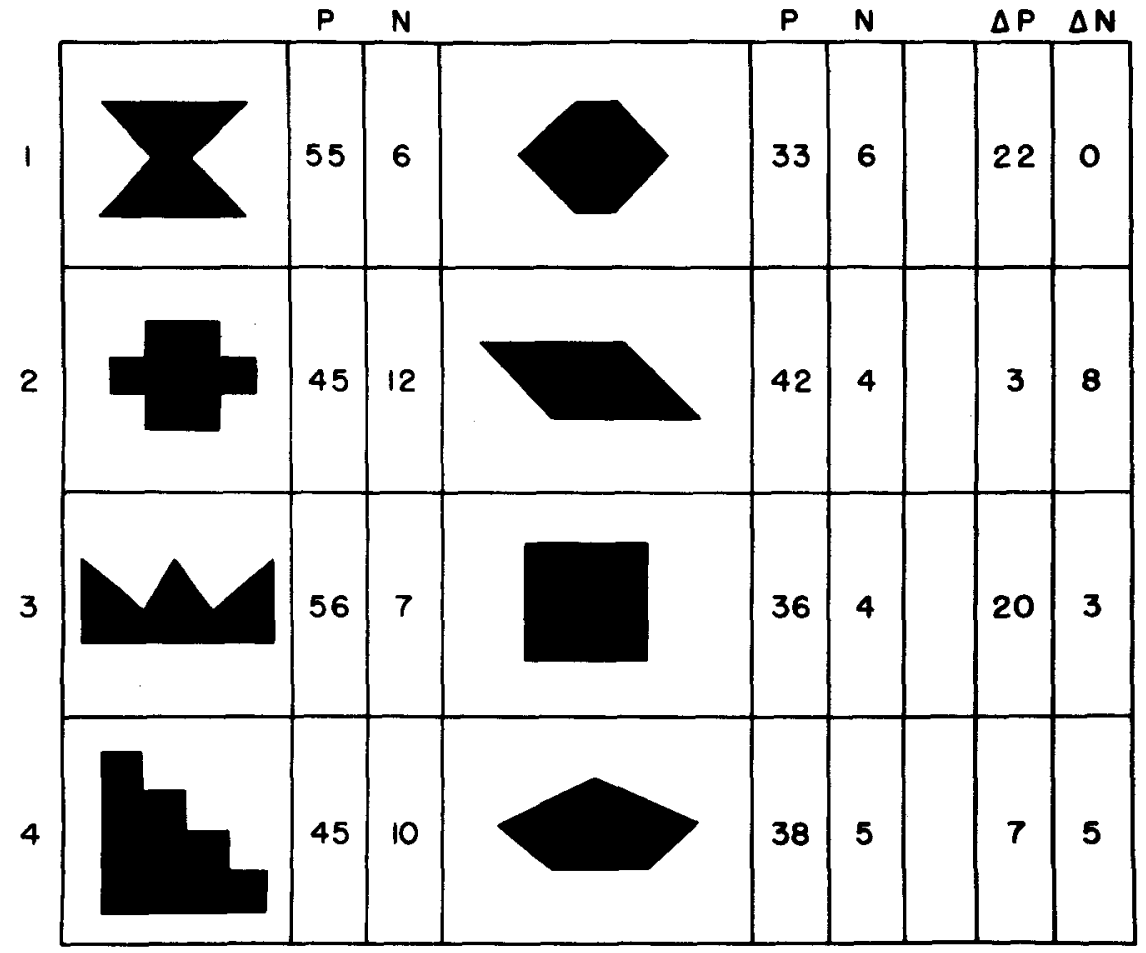

Fig. 3. Pairs of shapes used in the generalization tests; $P$ denotes the perimeter of each shape in centimeters, and $N$ denotes the number of sides. 
trials in Test 2; the Ss trained to select thyopen figure chose the open shape on $53 \%$ of the trials in Test 1 , and on $61 \%$ of the trials in Test 2. The difference between the two groups on Preference Test 2 is significant at the $0.1 \%$ level of confidence $(F=23.2, \mathrm{df}$ $=1 / 62$ ).

Transfer and Retention Tests

Mean errors to criterion for the consistent and inconsistent groups trained on the two stimulus pairs in counterbalanced order on the learning, transfer, and retention tasks are given in Fig. 2, which shows that the groups were matched adequately in terms of initial learning performance and that the inconsistent groups were substantially inferior to the consistent groups in performance on the transfer and retention tasks. Analysis of variance indicates that the difference between the consistent and inconsistent groups is significant at the $0.1 \%$ level on both the transfer $(F=$ 28.1 , df $=1 / 56$ ) and retention problems $(F=14.5, \mathrm{df}=1 / 56)$. There was no significant difference in performance between the groups trained on the circle vs $U$ and the triangle vs I discriminations or between the groups rewarded for choosing the open or closed shape on the learning, transfer, or retention phases of the experiment.

\section{Relationship between Preference} and Learning Scores

Product moment correlations were computed between the degree of preference each cat exhibited in Preference Tests 1 and 2 for the stimulus that was subsequently reinforced in the learning and transfer phases and for errors to criterion in discrimination learning. The $r$ between preferences on Test 1 and errors to criterion in original learning was -.42 , and the correlation between preferences on Test 2 and errors on the subsequent transfer problem was -68 . Both these values of $r$ are significant at well beyond the $1 \%$ level of confidence with 64 cases.

\section{Generalization Tests}

The shapes used in the generalization tests were equated in respect to size; all were $80 \mathrm{~cm}^{2}$ in area, as were the training shapes. The four pairs of test figures, illustrated in Fig. 3, were selected to provide a substantial difference in perimeter $(P)$ with little or no difference in number of sides (N), as in Pairs 1 and 3 , or to provide a rather large difference in number of sides and a relatively small difference in perimeter, as in Pairs 2 and 4. The two dimensions were opposed in this fashion in the hope that some information might be
INCONSISTENT

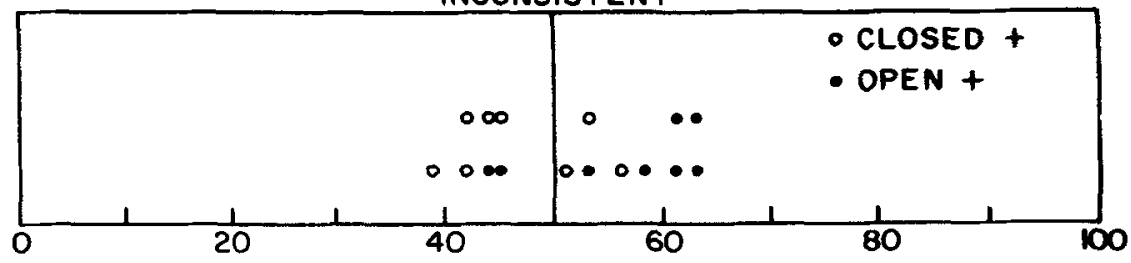

CONSISTENT

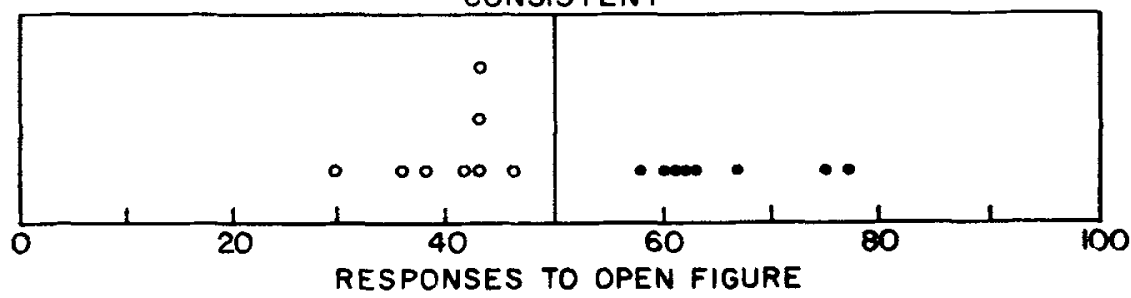

Fig. 4. Percentage of responses to the open figure by individual cats on four 25-trial generalization tests (100 trials).

obtained concerning their relative importance as determinants of shape classification by cats. The results of the generalization tests are summarized in Table 1 and Fig. 4. Table 1 shows the mean percentage of responses to the test figure that were appropriate in terms of the contingencies that prevailed on the intercurrent training trials by the cats that had been consistently or inconsistently reinforced in prior training; it also indicates the number of cats in the consistent and inconsistent groups (both $\mathrm{Ns}=16$ ) that displayed significant appropriate $(+)$ or inappropriate $(-)$ preferences $(\geqslant 18 / 25$ or $\leqslant 7 / 25$, respectively) on each pair of test figures. The consistent group manifested a stronger preference than the inconsistent group on every pair except 3 , on which both groups performed at chance, and none of the consistent cats displayed a significant preference for the stimulus that was inappropriate in terms of current reinforcement contingencies, while such paradoxical preferences were seen in six tests with members of the inconsistent group. The mean scores for the consistent group suggest differences in the number of sides between the two figures in a pair may be more important than differences in perimeter to cats, since the group's preference scores were substantially higher on Pairs 2 and 4 than on Pairs 1 and 3.

The overall results of generalization testing are summarized in Fig. 4, which shows the number of responses to the open figure on $\mathbf{1 0 0}$ test trials by individual cats that had been consistently or inconsistently reinforced in prior training. All of the cats in the consistent groups that had been trained to choose the open or closed figure responded appropriately by selecting the open figure more or less often than chance expectancy. Not only is there no overlap between the two classes in these groups, but there is a fairly wide gap between them. The inconsistent groups did not yield such orderly results. Some individuals did not select the open or closed test figures in conformity with the reinforcement contingencies prevailing on intercurrent training trials; consequently, the distribution of scores for the Ss rewarded differentially for responding to open and closed figures overlapped substantially. Analysis of variance showed that the difference between the consistent and inconsistent groups was significant at the $1 \%$ level of confidence $(F=10.9, \mathrm{df}=1 / 66)$, separate analysis of variance on the scores for the consistent group revealed, however, that test pairs was a significant source of variance, at the $5 \%$ level of confidence $(F=3.74$, $\mathrm{df}=$ $3 / 33$ ); this separate analysis seems justified in view of the high variability of performance by $S s$ in the inconsistent group.

Table 1

Performance of Consistent (C) and Inconaistent (I) Groups on Preference Tests

\begin{tabular}{|c|c|c|c|c|c|c|}
\hline \multirow{3}{*}{$\begin{array}{l}\text { Test } \\
\text { Pairs }\end{array}$} & \multirow{2}{*}{\multicolumn{2}{|c|}{$\begin{array}{c}\text { Percent } \\
\text { Appropriate } \\
\text { Responses } \\
\end{array}$}} & \multicolumn{4}{|c|}{$\begin{array}{c}\text { Significant } \\
\text { Preferences }\end{array}$} \\
\hline & & & \multicolumn{2}{|c|}{$\mathrm{C}$} & \multicolumn{2}{|c|}{$I$} \\
\hline & $\mathbf{C}$ & I & + & - & + & - \\
\hline 1 & $61 *$ & 51 & 4 & 0 & 4 & 3 \\
\hline 2 & $68 *$ & 57 & 7 & 0 & 5 & 2 \\
\hline 3 & 51 & 54 & 2 & 0 & 2 & $\overline{1}$ \\
\hline 4 & $70^{*}$ & 57 & 8 & 0 & 3 & 0 \\
\hline
\end{tabular}

* $\chi^{2}$ significant at $1 \%$ level of confidence while neither test pairs nor days was a significant source of variance. A 


\section{DISCUSSION}

Naive cats do not have a strong preference for open or closed figures. After discrimination training with differential reward for responses to open or closed shapes, however, cats tend to prefer an unfamiliar closed or open figure, depending upon which class had been reinforced in prior training. Cats trained to select the open or closed figure in both learning and transfer problems learn the transfer task much more quickly than do animals that must reverse their responses to open and closed figures between the initial learning and transfer tasks. Animals that receive inconsistent training in learning and transfer problems are also inferior in retention of the initial discrimination habit and fail to respond to the most recently rewarded stimulus class as often as consistently trained cats on postcriterion generalization tests.

These findings consistently support the view that cats categorize simple geometrical shapes as open or closed. It should be recognized, however, that this tendency in cats is not so strong as to override the effects of specific training with differential reinforcement. Although the inconsistent groups were inferior to the consistent groups in retention testing, they showed, as may be seen in Fig. 2, quite substantial savings in relearning their original discrimination, in spite of interpolated training to choose alternate values on the open-closed dimension.

It must also be recognized that we know little about the cues upon which cats classify shapes as open and closed. The results of the generalization tests indicate that, for the current sample of figures, differences in number of sides are more consequential than differences in perimeter. The generality of this finding remains to be determined, as does the significance of several other parameters that may influence the categorization of shapes by cats: differences in height, width, dispersion, number of reentrants, distinctive features, etc.

In spite of these reservations, it is gratifying to note that the results of this experiment are quite similar to those obtained in previous studies of shape discrimination by cats that used different procedures and paradigms (Derdzinski \& Warren, 1969; Warren \& McGonigle, 1969b). The results of this and our previous experiments with cats are all in good qualitative agreement with studies that indicate that octopus (Sutherland, 1960), fish (Sutherland, 1961), and rats (Sutherland \& Carr, 1962) discriminate visual forms in terms of an open-closed dimension.

Although most of the cats did not manifest strong preferences for the open and closed figures on Preference Tests 1 and 2, their scores on these tests were significantly correlated with errors to criterion in subsequent acquisition an;transfer learning. These observations indicate that preference tests with nondifferential reward are not inevitably insensitive measures of what animals have learned in prior training, as has been suggested in previous papers by Mumma and Warren (1968) and by Warren and McGonigle (1969a). Unfortunately, the present experiment does not permit us to make any unequivocal inferences concerning the conditions under which preference scores yield valid predictions of subsequent learning performance.

\section{REFERENCES}

DERDZINSKI, D. \& WARREN, J. M Perimeter complexity and form discrimination learning by cats. Journal of Comparative \& Physiological Psychology, 1969, 68, 407-411.

MUMMA, R. \& WARREN, J. M. Two-cue discrimination learning by cats. Journal of Comparative \& Physiological Psychology, 1968, 66, 116-121.

SUTHERLAND, N. S. Visual discrimination of shape by Octopus: Open and closed forms. Journal of Comparative \& Physiological Psychology, 1960, 53, 104-112.

SUTHERLAND, N. S. The methods and findings of experiments on the visual discrimination of shape by animals. Experimental Psychology Society Monographs, 1961, 1, 1-68.

SUTHERLAND, N.' S., \& CARR, A. E $V$ isual discrimination of open and closed shapes by rats: II. Transfer tests. Psychology, 1962, 14, 140-156.

WARREN, J. M., \& MCGONIGLE, B Effects of differential and nondifferential reinforcement on generalization test performance by cats. Journal of Comparative \& Physiological Psychology, 1969a, 69, 709-712.

WARREN, J. M., \& MeGONIGLE, B. Perimeter, complexity and generalization of a form discrimination by cats. Psychonomic Science, 1969b, 17, 16-17.

(Received for publication May 12, 1972; revision received July 21,1972 .) 